\title{
Structural design and motion analysis of Hexapod Bionic walking
}

\section{device}

\author{
Zhu Zhifang ${ }^{12}$ \\ 1.Jiangxi Province Key Laboratory of precision drive and control,Nanchang,330099,China \\ 2.Nanchang Institute of Technology,Nanchang,330099,China
}

Key words: Bionics; foot walking device; structure design; motion simulation

\begin{abstract}
Based on the principle of bionics, this paper designs a hexapod walking device by using a crank rocker mechanism. The designed structure can perform some basic walking operations, such as forward and backward place, turn left, turn right and so on. Furthermore, the triangle gait principle of the hexapod walking device and its stability are analyzed, the key parts of the device dimensions are calculated in detail, and the simulation of the body center of mass motion curve is performed to test the prototype through the dynamic simulation software ADAMS. The results show that the hexapod walking motion device is stable, with good mobility, and meets the design requirements.
\end{abstract}

\section{Introduction}

With the development of science and technology, for the human cannot reach place and may endanger the complex environment of human life are increasingly using mobile robot to complete, so can greatly avoid bad working environment of the harm to human body ${ }^{[1,2]}$. Mobile robot, the wheel or caterpillar mobile method is the most common way of moving, has been widely applied, its advantage is that move quickly, simple control, if mobile process road flat, relatively stable movement, the robot center of mass does not change ${ }^{[3,4]}$. But for complex irregular and uneven ground environment, the foot type mobile mode with wheeled and tracked mobile mode that the advantages of robots work in order to adapt to more and more complicated environment, foot type mobile robot research has become a striking in robotics research $^{[5]}$.

Based on the bionics principle, with hexapod outline insects is a bionic prototype, crank rocker mechanism as hexapod, and controlled by single chip microcomputer, consists of two small dc motors with gear box as driving element to design a kind of bionic hexapod walking device, and through the simulation software Adams is analyzed and the prototype test, results showed that the movement process smoothly.

\section{Overall structure design of Hexapod walking device}

In this paper, the design diagram of the hexapod walking device structure is shown in figure 1. The device structure including two parts: mechanical part and control part. Mechanical part is mainly composed of the body, two small dc motor, gear transmission mechanism, the six sets of four bar linkage ${ }^{[6]}$. Dc motor drive, the motor shaft position to spur gear, work through the motor output gear drive shaft rotation, thereby giving impetus to the movement of crankshaft movement to control the device.

Due to the same side of the foot joints by the crank shaft and the shaft coupling, so it can be controlled by adjusting the crankshaft Angle similarities and differences as well as the 
phase difference of each phase leg can achieve different gait, foot walking device is designed in accordance with the "four bar linkage", is the crank and rocker mechanism type used. In the crank rotates, the robot's foot to wiggle the elliptical shape, so the movement is similar to step when walking. Drive hexapod by six of the crank to strictly consistent, three foot touches the ground at the same time, the same crank to be practical. At this point, the three legs of the crank from the ground to rotate 180 degrees, correct installation of the crank is related to the robot walking pace to the right.

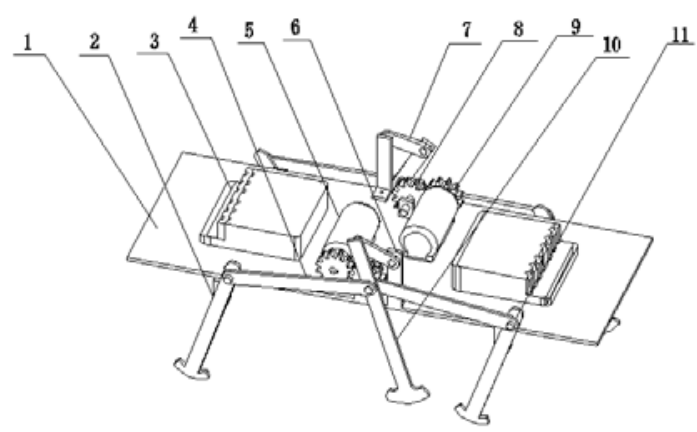

1.Base plate; 2. Your front foot; 3.Controller; 4. Connecting rod; 5. Motor; 6. Vertical bar; 7. Cross bar; 8.

Drive gear; 9.Output gear; 10. Her feet; 11. Hind feet

Fig.1. Structure of hexapod bionic walking device

\subsection{Foot design of walking device}

Hexapod should be designed according to the size of the device as a whole, the key is to set foot should not be too big or too small, if the foot is too small, it will not be able to jack the whole jacking device ${ }^{[7]}$; if the foot is too large, feet walking device will have to overcome a lot of work, so the foot of this device set should be modest, should be designed in between 20 to 40 degrees. As shown in figure 2 is the prototype of hexapod model diagram.

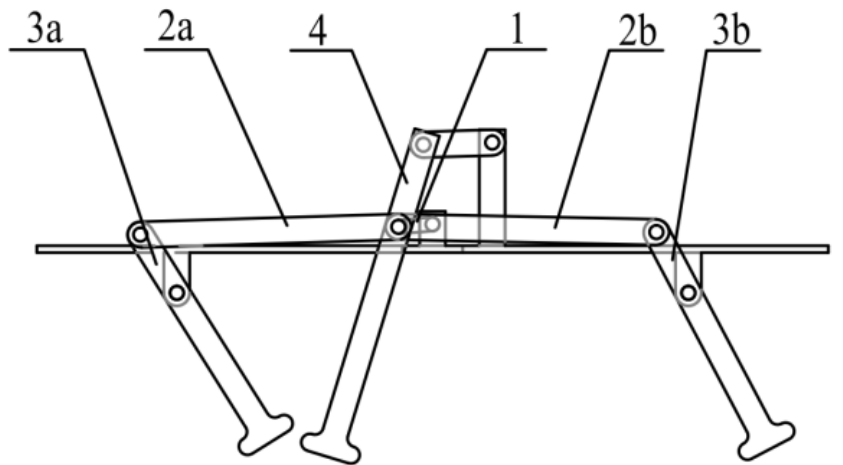

1.Crankshaft;2. Rail;3. different turning points in the upper part; 4. feet rod rotation point in upper part

Fig.2. Hexapod structure

Crankshaft length is set $a$, two rail length for $b$, upper center turning point length for different $e$, top length of foot pole center rotating point in $g$.

When the crankshaft movement to the highs and lows, three feet parallel and vertical to the ground, so the top require different rod center on a level with the crankshaft and the both feet and the horizontal distance between the foot in $\mathrm{f}=\sqrt{b^{2}-a^{2}}$, forefoot rod rotation center and the crankshaft center distance of: $\sqrt{b^{2}-a^{2}+e^{2}}$

Set the initial position, the front foot pole and rotation center and the Angle between the crankshaft center for $\alpha$, the current rod rotation $\theta$ to maximum pendulum foot, foot on the 
crankshaft and rod in the same line, the front foot pole and rotation center and the Angle between the crankshaft center for $\beta$.

$$
\cos \alpha=\frac{e}{\sqrt{b^{2}-a^{2}+e^{2}}}
$$

So: $\quad a=\arccos \frac{e}{\sqrt{b^{2}-a^{2}+e^{2}}}$

Rod rotation to maximum pendulum is current the feet, the crankshaft and the cross bar on the same line, set the front foot pole and rotation center and the Angle between the crankshaft center for $\beta$, on the front foot endpoint with the crankshaft center distance of $b+a$, based on the cosine theorem.

$$
\begin{aligned}
& \cos \beta=\frac{e^{2}+b^{2}-a^{2}+e^{2}-(a+b)^{2}}{2 e \sqrt{b^{2}-a^{2}+e^{2}}}=\frac{e^{2}-a^{2}-a b}{e \sqrt{b^{2}-a^{2}+e^{2}}} \\
& \text { So: } \beta=\arccos \frac{e^{2}-a^{2}-a b}{e \sqrt{b^{2}-a^{2}+e^{2}}}
\end{aligned}
$$

Both feet set foot is largest for:

$$
\theta=\beta-\alpha
$$

Through the above (1), (2) and (3) formula can even draw a different solution set foot is largest, the results calculated formula is as follows:

$$
\theta=\arccos \frac{e^{2}-a^{2}-a b}{e \sqrt{b^{2}-a^{2}+e^{2}}}-\arccos \frac{e}{{\sqrt{b^{2}-a^{2}+e}}^{2}}
$$

\section{Analysis of Hexapod bionic gait}

The hexapod bionic robot is three feet on one side of the pole synchronous motion, on both sides of different motor drive control through two groups of foot pole movement respectively ${ }^{[8]}$, the both feet on one side of the fixed triangle formed and on the other side of the foot, two groups of triangular interaction can realize the hexapod bionic robot walk straight, turn and lock. The bar top trajectory is shown in the following figure.

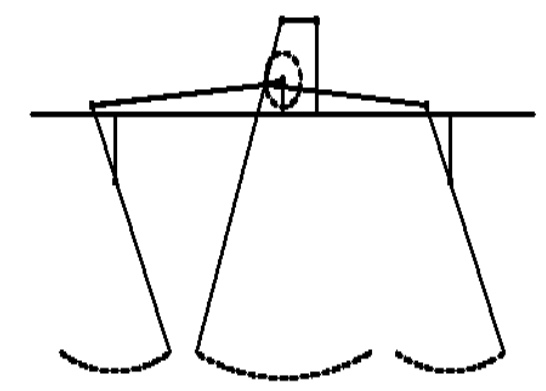

Fig.3. Foot bar at the top of track

\subsection{Foot pole movement process analysis}

Foot pole movement process is shown in figure 4 : 

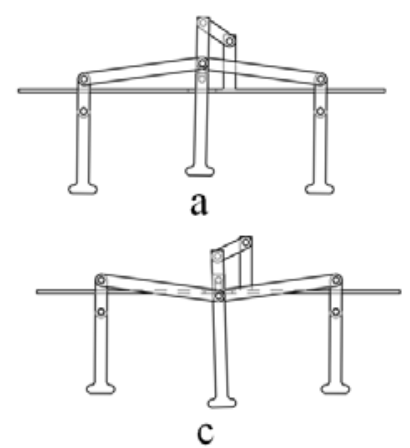
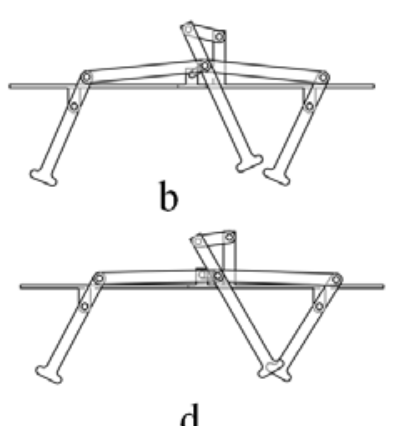

d

Fig.4. Foot pole movement process

(1)When the initial position, the crankshaft is perpendicular to the ground, three feet parallel and both feet on the ground, carried to the highest position in the feet.

(2) With connecting rod crank shaft to rotate counterclockwise to 2 on the same line, before and after the bar clockwise to maximum set foot.

(3) Crankshaft counterclockwise rotation, half a circle at this point in the foot with the other measurement of vertical ground common to jacking device at different rotary to parallel to the pole in different times.

(4) Crank shaft rotate counterclockwise to the connecting rod on the same line, different rotate counterclockwise to maximum pendulum feet, foot in clockwise to the ground.

(5) Crankshaft counterclockwise rotation a week to the origin, the entire unit to complete a cycle of walking.

\subsection{Based on Admas centroid dynamics analysis}

In order to research center of mass of the movement of walking device, application of center of mass for the dynamic simulation of Adams software, the simulation results are shown in figure 5 and figure 6 , the curve for the center of mass in the case of vertical velocity changes:

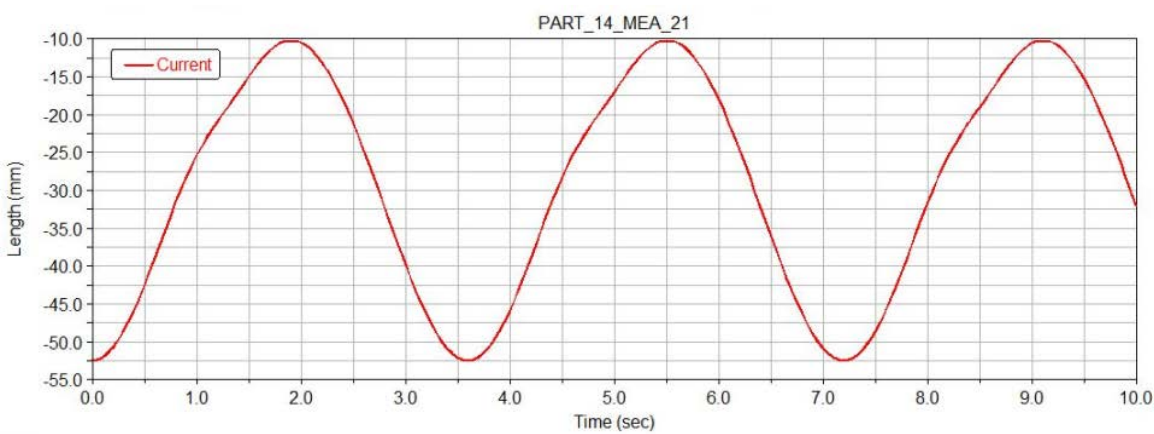

Fig.5. The centroid position change curve

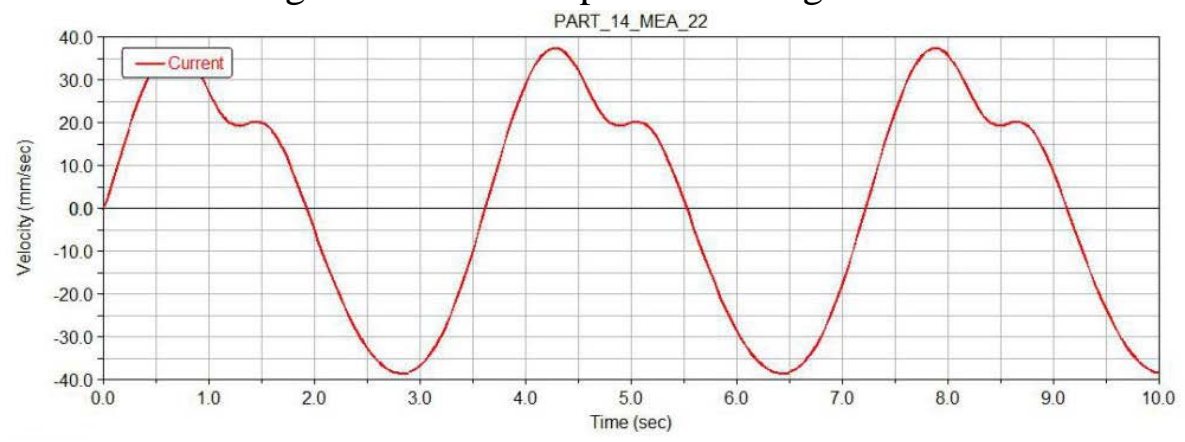

Fig.6. The center of mass velocity curve

Can be seen from the center of mass position and velocity curve, the center of mass 
center of gravity in the vertical direction of fluctuating $21 \mathrm{~mm}$, maximum amplitude of the mass center around before and after the location of the change to near zero. And the change of the center of mass velocity in the vertical direction is basically in accordance with the law in a changing, the center of mass movement speed is about $39 \mathrm{~mm} / \mathrm{s}$, the biggest integral curve is very smooth, basically the fluctuation is not big, uniform speed change, it can be seen walking device running very smooth, no obvious fluctuation.

\section{Prototype test}

The bionic analysis robot physical diagram is shown in figure 7. Produced by the body USES the high strength aluminum alloy material, six legs are made of 45 steel, which could greatly improve the overall strength. As shown in figure 2 of Hexapod robot is located in the median, the overall length and $400 \mathrm{~mm}, 160 \mathrm{~mm}$ wide, $200 \mathrm{~mm}$ high, middle legs and back before the site distance is $170 \mathrm{~mm}$, the overall quality of $2.25 \mathrm{~kg}$.

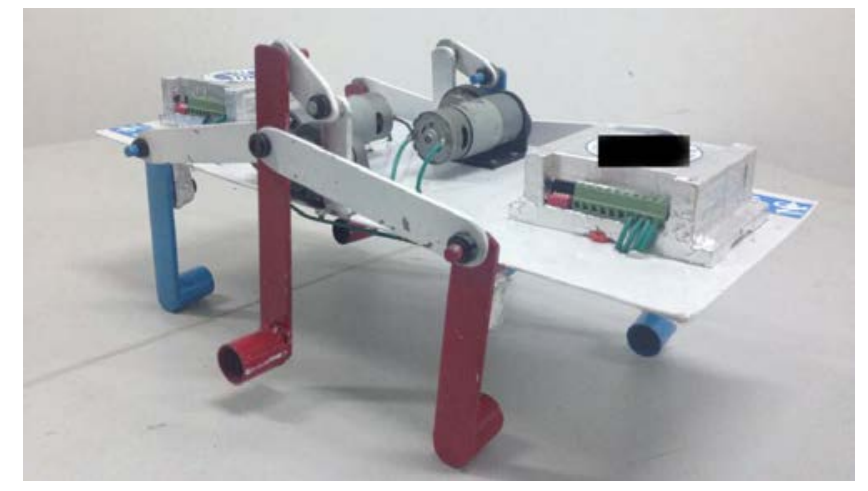

Fig.7. Physical objects of hexapod bionic robot

\section{Conclusion}

In view of the existing hexapod device structure design, hexapod trajectory problems such as unclear, based on bionics principle, solves the existing walking device structure design, trajectory analysis problems such as unclear, mainly for the general structure of the walking device design, main components of the motion simulation and prototype production, etc.

Walking through the plant overall structure design, the use of six bar linkage for bionic movement, the design principle of hexapod, obtained the gait principle; Parameters for the key parts design and application of Adams software modeling and simulation, the movement of mass center of moving devices, dynamic simulation curves, the results show that the device reasonable overall structure design, the center of mass movement smoothly, and finally made a prototype experiment, effect is good, has reached the expected design purpose.

\section{Acknowledgement}

In this paper, the research was sponsored by the Youth Science Fund of Nanchang Institute of Technology(Grant No.2014KJ012)And 2015 Outstanding graduation thesis of Nanchang Institute of Technology.

\section{References}


[1] Waldron K J, Vohnout V J, Pery A, et al.Configuration design of the adaptive suspension vehicle [J] .International Journal of Robotics Research, 1984, 3( 2) : 37-48.

[2] Jan Albiez, Karstan Berns. Biological Inspired Walking-How Much Nature Do We Need? [C] .Climbing and Walking Robots:Part III.Berlin Heidelberg: Springer, 2005.

[3] Jiang Shuhai.Development Status of Forestry Robot[J].Journal of Northeast Forestry University,2009,37( 12) : 95-97.

[4] Hu Jing,Jiang Shuhai.Robot Multi-sensor Information fusion technology [C] . Bankok, Thailand: The 11th International Conference on Intelligent Technologies,2010.

[5] Chen Li. Research on Control and Target Detection System for Hexapod Bionic Disaster Relief Robot [D] .NanJing: Nanjing forestry university,2011.

[6] Tang Jingjing. Research on Virtual Prototyping for Disaster Rescue Hexapod Bio-Robot[D]. NanJing: Nanjing forestry university,2011.

[7] Sun Pei. Research on Control System for Hexapod Bionic Robot[D]. NanJing: Nanjing forestry university,2010.

[8] Bernsk. Internet address for the walking machines catalogues [J] . 2011,(07):21- 25. 